\title{
Polarimetric Observations of Active Cool Binaries
}

\author{
R.H. Koch ${ }^{1}$, N.M. Elias ${ }^{1}$, B.D. Holenstein ${ }^{1}$, V. Piirola ${ }^{2}$, \\ F. Scaltriti ${ }^{3}$, and G.V. Coyne ${ }^{4}$ \\ ${ }^{1}$ University of Pennsylvania, Philadelphia, U.S.A. \\ ${ }^{2}$ Observatory and Astrophysics Laboratory, Helsinki, Finland \\ ${ }^{3}$ Osservatorio Astronomico di Torino, Pino Torinese, Italia \\ ${ }^{4}$ Vatican Observatory, Città del Vaticano
}

\section{Introduction}

For a sample of RS CVn binaries Busso and collaborators (Busso et al., 1987, 1988; Busso and Scaltriti, 1990) compared the observed energy distribution from the UV to the near-IR (and to IRAS bandpasses for some objects) with those calculated from the known radii and spectral types of the stellar components. About $25 \%$ of the sample gave evidence of an IR excess up to $0.7 \mathrm{mag}$, generally starting from the I-band. The excess is significantly larger than observational error. These same authors ascribe the excess to a thin dust shell surrounding the binary. Using the "dirty silicates" model by Jones and Merrill (1976) they find for the shell a temperature range of $1500 \mathrm{~K}$ to $2000 \mathrm{~K}$, an optical depth of about 0.01 at 10 micrometers in the shell, and a distance of the shell from the stars to be typically about 100 times the radius of the larger star.

Multi-band polarimetry represents a powerful tool for investigating circumstellar scattering envelopes. However, it has not been commonly applied to RS CVn binaries. Published data indicate that the intrinsic polarization is small, although Pfeiffer (1979) and Liu and Tan (1987) have found values in excess of $0.3 \%$ for certain binaries at unpredictable times. Piirola (in Weiler et al., 1978) observed two RS CVn's (UX Ari and HR 1099) and found very small values of linear polarization $(p<0.02 \%)$ in white light.

Both general arguments (e.g. Popper and Ulrich, 1977) concerning the evolutionary states of RS CVn's and observations of individual binaries indicate mass loss by winds and the presence of circumstellar and circumbinary envelopes of gas and dust in the systems. Since non-spherical concentration of neutral H, plasmas, and dust can all give rise to significant levels of polarization, we started a collaboration in 1989 to monitor selected RS CVn's polarimetrically from the U-band into the near-IR. 


\section{The observational campaign and instrumentation}

Multiband polarimetry was performed in 1989-1990 at four sites: (1) at Kitt Peak the 90-inch telescope of Steward Observatory was used together with the 'twoholer' polarimeter of Gary Schmidt; (2) at Pennsylvania the 0.72-m Cassegrain reflector was used together with a photoelastic polarimeter described by Koch et al. (1985); (3) at La Palma the observations were made with the 2.56-m Nordic telescope by using the simultaneous five colour (UBVRI) polarimeter built in Turku Observatory (Piirola, 1973; Korhonen et al., 1984); (4) at Torino the 1.05-m astrometric reflector fed the 5-channel (UBVRI) simultaneous polarimeter recently constructed at the Institute according to the original design by Piirola (1973, 1988). Some details of this polarimeter can be found in Scaltriti et al. (1989).

\section{Results and discussion}

In general, it may be noticed that the linear polarization values are typically small. This is consistent with the published results noted above. In order to perform a more comprehensive discussion, we divide our sample results into three sub-groups according to the amount of phase coverage of the Keplerian cycles.

(a) Single-phase measures. In B the measured polarizations for Zeta And and WY Cnc are consistent with zero $( \pm 0.02 \%)$. The $\mathrm{B}, \mathrm{V}$ and $\mathrm{R}$ measures for RU Cnc and WY Cnc appear significant at the $0.05 \%$ level. The polarization spectrum of RU Cnc is flat within the errors of measurement. (b) Two phases sampled. Each of the stars CQ Aur, RZ Cnc, VV Mon, and AR Mon shows significant polarization (0.1$0.2 \%$ ). The polarization wavelength dependence for these systems indicates that within 3-sigma, they are consistent with the wavelength dependence of interstellar polarization. (c) At least 5 phases sampled. For GK Hya there is marginal evidence of p-variability in the R and I bands. Also for AR Psc there are hints of p-variability in the $B, R$, and I bandpasses but these would have to be confirmed by more observations.

The best phase coverage is that for UX Ari in the B,V, and R bands and for II Peg in the V-band. The linear polarization may be phase locked. It is tempting to try to interpret this pattern as being due to a scattering envelope asymmetrically distributed with respect to the low-inclination orbital plane as in the model of Brown et al. (1978). The mean wavelength dependence of the linear polarization compiled from the Torino data suggests that electron scattering is not the cause of the intrinsic polarization, since the amount of polarization decreases toward longer wavelengths. A similar tendency is seen also for GK Hya, UV Psc, and AR Psc (see Fig. 1), indicating small dust particles as a possible source of the intrinsic polarization.

With respect to the main objective of the observational program our flat spectrum (interstellar type) sample contains two pairs with an IR-excess (RU Cnc, VV Mon), two pairs without the excess (CQ Aur and RZ Cnc) and one with uncertain excess (AR Mon). The binaries showing indications of polarization by circumstellar dust (Fig. 1) contain two pairs with an IR-excess (GK Hya, II Peg), 


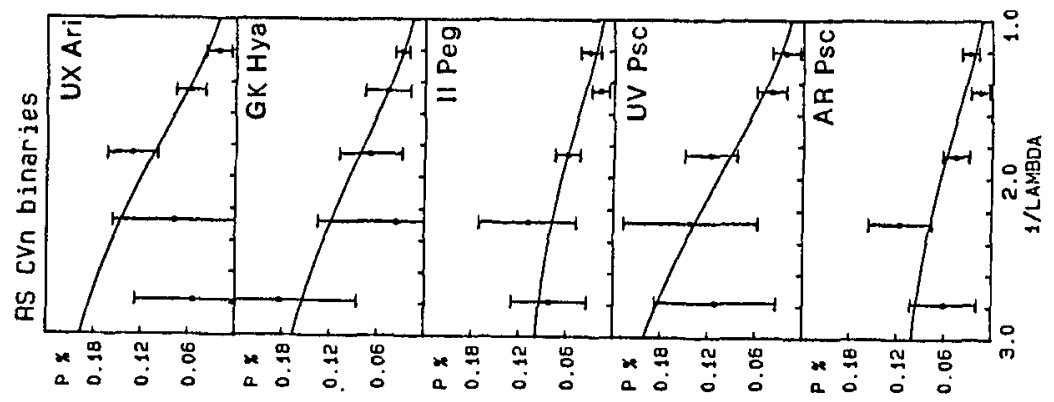

Fig. 1. The wavelength dependence of the mean polarization for five RS CVn systems. There is a tendency of smaller polarization toward longer wavelengths.

two without (UX Ari, AR Psc) and one with uncertain excess (UV Psc). In addition, WY Cnc, which has the IR excess but was observed only in two passbands, shows marginal indication of larger polarization towards longer wavelengths $(p(B)=0.027 \pm 0.021 \% ; p(R)=0.060 \pm 0.017 \%)$.

The polarization of some of these binaries may be variable in the long term and we plan to continue monitoring some of these systems. In particular, II Peg will undoubtedly repay continuous linear polarimetric monitoring, a conclusion hardly surprising in view of its activity. Moreover, the suggestive linear results for UX Ari and GK Hya indicate that detailed monitoring of the polarization with phase and wavelength is necessary.

\section{References}

Brown, J.C., McLean, I.S., and Emslie, A.G.: 1978, Astron. Astrophys. 68, 4 i3

Busso, M., Scaltriti, F., Persi, P., Robberto, M., and Silvestro, G.: 1987, Astron. Astrophys. 183, 83

Busso, M., Scaltriti, F., Persi, P., Ferrari-Toniolo, M., and Origlia, L.: 1988, Mon. Not. R. astr. Soc. 234, 445

Busso, M., and Scaltriti, F.: 1990, in preparation.

Jones, T.W., and Merrill, K.M.: 1976, Astrophys. J. 209, 509.

Koch, R.H., Hrivnak, B.J., Bradstreet, D.H., Blitzstein, W., Pfeiffer, R.J., and Perry, P.M.: 1985, Astrophys. J. 288, 731

Korhonen, T., Piirola, V., and Reiz, A.: 1984, The ESO Messenger, December 1984

Liu Xuefu and Tan Huisong: 1987, Acta Astr. Sinica 28, 139.

Pfeiffer, R.J.: 1979, Astrophys. J, 232, 181.

Piirola, V.: 1973, Astron. Astrophys. 27, 383.

Piirola, V.: 1988, in Polarized Radiation of Circumstellar Origin, eds. G.V. Coyne et al., Univ. of Arizona Press, p. 735.

Popper, D.M., and Ulrich, R.K.: 1977, Astrophys. J. Letters 212, L131.

Scaltriti, F., Piirola, V., Cellino, A., Anderlucci, E., Corcione, L., Massone, G., Racioppi, F.: 1989, Mem. S.A.It. 60, 243.

Weiler, E.J., Owen, F.N., Bopp, B.W., Schmitz, M., Hall, D.S., Fraquelli, D.A., Piirola. V., Ryle, M., and Gibson, D.M.: 1978, Astrophys. J. 225, 919. 